\title{
GeSELLSCHAFTLICHE DeTERMINANTEN PRODUKTIVEN ALTERNS IN EUROPA
}

Karsten Hank \& Stephanie Stuck 


\title{
Gesellschaftliche Determinanten produktiven Alterns in Europa
}

\author{
Karsten Hank $k^{\mathrm{a}} \&$ Stephanie Stuck ${ }^{\mathrm{b}}$
}

\begin{abstract}
Oktober 2007
Zusammenfassung: Ziel des vorliegenden Beitrages ist es, $\mathrm{zu}$ einem besseren Verständnis der gesellschaftlichen Determinanten produktiven Alterns jenseits der Erwerbstätigkeit beizutragen. Auf Basis von Daten des Survey of Health, Ageing and Retirement in Europe (SHARE) und unter Verwendung logistischer Mehrebenenmodelle zeigen wir, dass ein substantieller Teil der Varianz zwischen den elf hier betrachteten Ländern hinsichtlich des privaten Engagements in den Bereichen Ehrenamt, Netzwerkhilfe und Pflege auf Unterschiede in der Bevölkerungskomposition zurück geführt werden kann. Darüber hinaus tragen auch die drei von uns untersuchten Makroindikatoren erheblich zur Erklärung des Varianzanteils auf der Länderebene bei. Während gesellschaftliche Altersbilder jedoch keinen signifikanten Zusammenhang mit ehrenamtlichem Engagement, Netzwerkhilfe und Pflege aufweisen, werden unsere Hypothesen bezüglich eines positiven Zusammenhangs zwischen politischer und religiöser Freiheit sowie stärkerem wohlfahrtsstaatlichem Engagement und der individuellen Neigung Älterer zur Ausübung informeller produktiver Tätigkeiten weitgehend bestätigt. Aus den vorgestellten empirischen Befunden werden abschließend sozialpolitisch und sozialwissenschaftlich relevante Schlussfolgerungen für die Aktivierung bislang ungenutzter produktiver Potenziale in der älteren Bevölkerung gezogen.
\end{abstract}

Abstract: This paper aims to contribute to improving our understanding of the societal determinants of productive ageing beyond formal employment. Based on data from the Survey of Health, Ageing and Retirement in Europe (SHARE) and applying logistic multilevel models, our analysis shows that a substantial portion of the variance between the eleven countries in our study with regard to private engagement in volunteering, informal helping, and caring is due to differences in population composition. In addition, the three macro-level indicators in our study contribute to a further substantial reduction in the between-country variance. While societal images of ageing do not bear a statistically significant association with volunteer work, informal help, or care, our hypotheses concerning a positive relationship between greater political and religious freedom as well as welfare state services and older individuals' propensity to engage in informal productive activities are largely confirmed. We close with conclusions relevant for social policy and social science, regarding the activation of so far unused productive potentials in the elder population.

\footnotetext{
${ }^{a}$ MEA - Universität Mannheim \& DIW Berlin.Email: hank@mea.uni-mannheim.de.

${ }^{\mathrm{b}}$ MEA - Universität Mannheim. Email: stuck@mea.uni-mannheim.de.
} 


\section{Einleitung}

Vor dem Hintergrund der rapiden Bevölkerungsalterung in Deutschland und anderen hochindustrialisierten Staaten hat das Konzept des ,produktiven Alterns' in den vergangenen Jahren erhebliche Aufmerksamkeit erfahren (z.B. Erlinghagen \& Hank 2007; Künemund 2005; Morrow-Howell et al. 2001). Die Betonung des produktiven Potentials älterer Menschen stellt eine positive Alternative $\mathrm{zu}$ bislang häufig dominierenden negativen Alter(n)sbildern dar (vgl. Kruse \& Schmitt 2005), wenngleich nicht immer Einigkeit darüber besteht, welche Tätigkeiten als produktiv zu betrachten sind (ein Überblick über verschiedene Definitionen gibt z.B. Caro 2007: Abschnitt 2.1).

Grundsätzlich kann die ,formelle’ Erwerbsarbeit von einer Vielzahl ,informeller' Tätigkeiten (wie z.B. dem Ehrenamt) unterschieden werden, für die kein Lohn gezahlt und keine Abgaben entrichtet werden (vgl. hierzu ausführlich Erlinghagen 2000). Empirische Untersuchungen zeigen, dass es sowohl bei der Erwerbsbeteiligung (z.B. Brugiavini et al. 2005) als auch hinsichtlich des informellen Engagements Älterer in verschiedenen Bereichen familiärer (z.B. Hank \& Buber 2007) und außerfamiliärer (z.B. Erlinghagen \& Hank 2006) Unterstützung erhebliche Unterschiede zwischen einzelnen Ländern bzw. Ländergruppen gibt. Bemerkenswert hierbei sind die deutlichen Hinweise auf eine Komplementarität der Beziehung zwischen verschiedenen Tätigkeiten, d.h. Engagement in einem Bereich - individuell und aggregiert auf der Länderebene - ist positiv mit Engagement in anderen Bereichen korreliert (vgl. Hank \& Stuck 2007; Stuck 2006). Während die Ursachen der im internationalen Vergleich stark variierenden Erwerbsquoten älterer Menschen bereits gut untersucht sind, und hier vor allem auf die Bedeutung der Ausgestaltung des Rentensystems hingewiesen wurde (z.B. Gruber \& Wise 2004; Kohli et al. 1991), ist bislang weitgehend ungeklärt geblieben, welche spezifischen gesellschaftlichen Determinanten für die unterschiedliche Beteiligung an informellen produktiven Tätigkeiten im höheren Lebensalter verantwortlich sind. 
Auf Basis vergleichbarer Mikrodaten des 2004 erstmals erhobenen ,Survey of Health, Ageing and Retirement in Europe' (SHARE) untersucht der vorliegende Beitrag die Bedeutung gesellschaftlicher Rahmenbedingungen in 11 Ländern für das Engagement von Männern und Frauen im Alter von 50 oder mehr Jahren in den Bereichen Ehrenamt, Netzwerkhilfe und (haushaltsexterner) Pflege. Im folgenden Abschnitt 2 werden zunächst sozial-kulturelle und institutionelle Rahmenbedingungen informeller Arbeit bzw. produktiven Alterns diskutiert. Anschließend stellen wir in Abschnitt 3 unsere Datenbasis und das methodische Vorgehen in dieser Untersuchung vor. Auf die Beschreibung unserer deskriptiven Befunde und multivariaten Regressionsergebnisse in Abschnitt 4 folgt schließlich ein ausführliches Fazit (Abschnitt 5).

\section{Sozial-kulturelle und institutionelle Rahmenbedingungen produktiven Alterns: Konzeptuelle Überlegungen und Hypothesen}

Die Literatur $\mathrm{zu}$ den gesellschaftlichen Rahmenbedingungen informeller Arbeit ist bislang eher spärlich und fokussiert in erster Linie auf den Bereich des ehrenamtlichen Engagements (vgl. den Überblick bei Hank et al. 2006: 9f.). Vor dem Hintergrund des hier im Mittelpunkt stehenden Aspektes des ,produktiven Alterns' ergänzen wir diese Literatur durch die Berücksichtigung der Bedeutung gesellschaftlicher Altersbilder für die Ausübung informeller Tätigkeiten (vgl. Hypothese 1). Darüber hinaus wird der Versuch unternommen, die Reichweite von Erklärungsansätzen aus der international vergleichenden Ehrenamtsforschung auf die Bereiche Netzwerkhilfe und informelle Pflege auszudehnen (vgl. Hypothese 2 und Hypothese 3). Im Folgenden formulieren wir hierzu drei Hypothesen und betten diese in den aktuellen Forschungsstand ein.

Hypothese 1: Die Beteiligung älterer Menschen an informeller Arbeit wird durch kollektive Leistungsanforderungen im Alter, Konventionen über Altersgrenzen für produktive Tätigkeiten und die Bedeutung von Produktivität bzw. Aktivität im jeweils 
dominierenden gesellschaftlichen Altersbild mitbestimmt. Altersbilder und -stereotypen werden sozial geprägt und wirken sich nachweislich auf individuelles Verhalten aus (z.B. Filipp \& Mayer 2005; Staudinger 2003: 35f.). Mit Blick auf unsere Fragestellung dürften insbesondere betriebliche Sicht- und Verhaltensweisen gegenüber älteren Erwerbspersonen (vgl. Bellmann et al. 2003; Roscigno et al. 2007) sowie die Ausgestaltung für die Erwerbsbeteiligung Älterer relevanter Arbeitsmarktinstitutionen, einschließlich des Sozialversicherungssystems (z.B. Kalina \& Knuth 2002), bedeutsam sein. So weist etwa Göckenjan (2007) in einer Untersuchung zum Wandel des Altersbildes in der Folge der deutschen Rentenreform von 1957 darauf hin, dass erst als unintendierte Folge der Einführung die Lebenshaltungskosten deckender Renten ,das Rentenalter' als eigenständige, sozialrechtlich formierte Lebensphase entstand, die bis heute in aller Regel das vollständige Ende des Arbeitslebens bedeutet und so wesentlich zum Entstehen eines Bildes des ,funktionslosen Alters' beigetragen hat. Ein solches, ursprünglich dem Bereich der Erwerbsarbeit entstammendes, , unproduktives’ Altersbild könnte sich auch negativ auf den Bereich der Nicht-Erwerbsarbeit auswirken. Unterstellt man eine solche Beziehung, wäre anzunehmen, dass eine hohe Erwerbsbeteiligung Älterer, die ein eher, produktives' Altersbild widerspiegelt, positiv mit der Wahrscheinlichkeit korreliert ist, dass ältere Menschen informelle Arbeit leisten (unabhängig vom individuellen Erwerbsstatus; vgl. hierzu die Untersuchungen von Erlinghagen, 2007a, sowie Mutchler et al., 2003, über den Zusammenhang von Renteneintritt und freiwilligem Engagement).

Hypothese 2: Produktives Altern jenseits der Erwerbsarbeit findet nicht unabhängig von der ,Kultur' informeller Arbeit in einer Gesellschaft statt. Diese wird in der Literatur vor allem mit Bezug auf das Ehrenamt im Rahmen von Konzepten wie jenem der ,Zivilgesellschaft' und des ,bürgerschaftlichen Engagements' diskutiert (z.B. Anheier \& Toepler 2002; Salamon et al. 2003). Auch wenn zivilgesellschaftliches Engagement - natürlich - zwischen Kohorten variieren kann, etwa als Folge unterschiedlicher Sozialisationserfahrungen (z.B. Goss 1999; Rotolo \& Wilson 2004), 
lassen sich dennoch langfristig wirkende, historisch gewachsene sozial-kulturelle Rahmenbedingungen identifizieren, die sich insbesondere im Hinblick auf die Ausübung ehrenamtlicher Tätigkeiten als wichtig erwiesen haben (z.B. Curtis et al. 2001; Smith \& Shen 2002). Hervorzuheben ist in diesem Zusammenhang vor allem die Rolle der religiösen Prägung und der Kontinuität demokratischer Strukturen in einer Gesellschaft, die sowohl das Ausmaß individueller Autonomie als auch relevante rechtliche Rahmenbedingungen (z.B. Vereinsrecht) beeinflussen. Es wird daher angenommen, dass ein hohes Maß an religiöser und politischer Freiheit sich positiv auf die Wahrscheinlichkeit auswirkt, ein Ehrenamt auszuüben. Angesichts des in früheren Untersuchungen beobachteten komplementären Zusammenhangs zwischen ehrenamtlichem Engagement und Netzwerkhilfe sowie informeller Pflege (vgl. Hank \& Stuck 2007) vermuten wir zudem auch einen - mindestens indirekten - positiven Zusammenhang zwischen Freiheit und Engagement in anderen Bereichen informeller Arbeit.

Hypothese 3: Beachtenswert ist darüber hinaus die - ebenfalls kulturell geprägte (vgl. Pfau-Effinger 2005) - Ausgestaltung wohlfahrtsstaatlicher Institutionen bzw. Regime, wie sie etwa klassisch bei Esping-Andersen (1990) beschrieben wurden (siehe auch Schmid 2002). Im Kontext der vorliegenden Arbeit geht es hier vor allem um das Verhältnis von Staat und formellen sowie informellen sozialen Netzwerken bei der Erfüllung gesellschaftlich notwendiger bzw. gewünschter Aufgaben. Grundsätzlich lassen sich drei denkbare Szenarien unterscheiden: (1) wohlfahrtsstaatliches Engagement untergräbt privates Engagement (,crowding-out' Hypothese); (2) wohlfahrtsstaatliches Engagement fördert privates Engagement (,crowding-in' Hypothese); (3) die Beziehung zwischen wohlfahrtsstaatlichem und privatem Engagement ist ambivalent, d.h. sie variiert mit der Organisation und dem Tätigkeitsbereich, in dem informelle Arbeit stattfindet. Der ,Nettoeffekt' wohlfahrtsstaatlicher Leistungen auf den Umfang nicht-marktlicher produktiver Tätigkeiten in einem Land würde dann nicht zuletzt von deren dominanter 
Organisationsform oder von deren schwerpunktmäßigen Inhalten abhängen (vgl. hierzu auch Kääriäinen \& Lehtonen 2006). So argumentiert etwa Janoski (zitiert in Curtis et al. 2001: 786f.), dass im liberalen Regimetyp fehlende Leistungen des Wohlfahrtsstaates insbesondere durch freiwilliges Engagement in kirchlichen Organisationen substituiert werden, während sozialdemokratische Regime die Mitgliedschaft in Gewerkschaften und politischen Organisationen explizit ermutigen, in dem sie diese z.B. finanziell unterstützen. Im ersten Fall hätte freiwilliges Engagement, in der Terminologie von Salamon \& Sokolowski (2003), hauptsächlich eine soziale Dienstleistungsfunktion (, service role'), während im zweiten Fall die ,expressive role' dominieren würde, bei der Leistungen in erster Linie in den Bereichen Kultur und Politik im weiteren Sinne erbracht werden. Neben dem Ehrenamt ließe sich auch für die Netzwerkhilfe und insbesondere für die Pflege argumentieren, dass privates Engagement zwar fehlende staatliche Unterstützung kompensieren könnte, dass aber andererseits wohlfahrtsstaatliches Engagement möglicherweise überhaupt erst die (infrastrukturellen) Voraussetzungen für Eigeninitiative schafft.

Während die ,crowding-out' Hypothese als empirisch weitgehend widerlegt betrachtet werden kann, finden sich in verschiedenen Studien Befunde, die als Hinweis auf die Existenz zumindest moderater ,crowding-in' Effekte interpretiert werden können (z.B. Motel-Klingebiel et al. 2005; Salamon \& Sokolowski 2003; van Oorschot \& Arts 2005). Wir nehmen daher an, dass ein höherer Anteil der öffentlichen Sozialausgaben am Bruttoinlandsprodukt positiv mit der Ausübung informeller produktiver Tätigkeiten im Alter korreliert ist.

\section{Daten und Methode}

\subsection{Der ,Survey of Health, Ageing and Retirement in Europe'}

Datenbasis unserer Untersuchung ist Release 2.0.1 des 2004 erstmals erhobenen ,Survey of Health, Ageing and Retirement in Europe' (SHARE; vgl. Börsch-Supan et 
al. 2005; Börsch-Supan \& Jürges 2005). Das Themenspektrum der Umfrage umfasst gleichermaßen die gesundheitliche, wirtschaftliche und soziale Lage der mehr als 30.000 Befragten, die eine repräsentative Stichprobe der Bevölkerung im Alter von 50 und mehr Jahren in Privathaushalten darstellen. Die hier verwendeten Daten stammen aus 11 Ländern (Belgien, Dänemark, Deutschland, Frankreich, Griechenland, Niederlande, Italien, Österreich, Schweden, Schweiz und Spanien), die das kulturelle, wirtschaftliche, soziale und institutionelle Spektrum Europas von Skandinavien bis zum Mittelmeer breit abdecken. - Deskriptive Informationen zur hier verwendeten Stichprobe finden sich in Tabelle 1.

[Tabelle 1 etwa hier einfügen]

\subsection{Operationalisierung der Variablen ${ }^{1}$}

Die Angaben der Befragten zu ehrenamtlichen Tätigkeiten, Netzwerkhilfe und Pflegetätigkeiten beziehen sich auf eine allgemeine Frage zu produktiven und konsumtiven Aktivitäten der Studienteilnehmer, bei der auch Mehrfachnennungen möglich waren. Die Frage lautet: „Bitte sehen Sie sich Karte 35 an. Haben Sie sich im letzten Monat an einer der dort aufgeführten Aktivitäten beteiligt?“ Die hier verwendeten Antwortkategorien sind:

\section{Ehrenamtliche Tätigkeit}

2. Betreuung eines kranken oder behinderten Erwachsenen

3. Hilfe für Familienmitglieder, für Freunde oder für Nachbarn

Mit Blick auf die Analyse ehrenamtlicher Tätigkeiten besteht ein wesentlicher Vorteil der SHARE-Daten darin, dass nicht die Mitgliedschaft in einer Freiwilligenorganisation (z.B. Curtis et al. 2001), sondern die aktive Ausübung eines Ehrenamtes im Monat vor

\footnotetext{
${ }^{1}$ Die Auswahl und Definition der Variablen für die vorliegende Untersuchung erfolgte in enger Anlehnung an Hank \& Stuck (2007).
} 
dem Interview erfragt wird. Obwohl Mitgliedschaft und Engagement hoch miteinander korreliert sind, führt eine rein formale Operationalisierung des Ehrenamts zu einer Überschätzung des tatsächlichen Engagements. Da Ehrenämter darüber hinaus oft unregelmäßig ausgeübt werden, und die retrospektiven Fragen anderer Untersuchungen sich häufig auf einen längeren Zeitraum (z.B. das vergangene Jahr) beziehen, ist davon auszugehen, dass unsere Ergebnisse eine vergleichsweise konservative Schätzung des ehrenamtlichen Engagements darstellen (vgl. auch Erlinghagen \& Hank 2006).

Bei der Interpretation der Angaben zur Pflege ist zu beachten, dass in einem der allgemeinen Aktivitätsfrage vorgelagerten Modul des SHARE-Fragebogens bereits detaillierte Fragen zu Pflegetätigkeiten und Hilfe innerhalb und außerhalb des Haushalts während der letzten 12 Monate vor dem Interview gestellt wurden. Befragte, die bereits in diesem Fragenblock Pflegetätigkeiten berichtet haben, könnten bei der von uns ausgewerteten Frage dazu tendiert haben, Pflege, die im letzten Monat erbracht wurde, nicht noch einmal gesondert $\mathrm{zu}$ erwähnen. Außerdem ist anzunehmen, dass Pflegeleistungen innerhalb des eigenen Haushalts hier deutlich untererfasst sind, da die anderen erfragten Aktivitäten eine Interpretation der unserer Analyse zu Grunde liegenden Frage in Richtung haushaltsexternern Engagements nahe legen.

Ausgehend von der grundlegenden Annahme, dass produktive Tätigkeiten nicht ohne den Einsatz individueller Ressourcen erbracht werden können (z.B. Tang 2006; Wilson \& Musick 1998), wurden die individuellen Kontrollvariablen ausgewählt. Als demographische Merkmale gehen Geschlecht, Alter (50-64, 65-74, 75 Jahre oder älter) und Partnerschaftsstatus (mit oder ohne Partner lebend) in die Untersuchung ein. Als Indikatoren für den sozio-ökonomischen Status der Befragten werden der Erwerbsstatus (erwerbstätig, nicht erwerbstätig, im Ruhestand) sowie der höchste Bildungsabschluss verwendet, wobei die Bildung der Befragten nach der ISCED 97 Klassifizierung kodiert und anschließend in drei Gruppen zusammengefasst wurde. Als niedrige Bildungsabschlüsse sind hier die ISCED Kategorien 0-2 definiert (in Deutschland: kein Schulabschluss, Haupt- oder Realschulabschluss, jeweils ohne Berufsausbildung), 
mittlere Abschlüsse entsprechen den ISCED Kategorien 3 und 4 (in Deutschland: Haupt- oder Realschule mit Berufsausbildung, Fachhochschulreife, Abitur) und die höchste Bildungskategorie fasst die ISCED Kategorien 5 und 6 zusammen (in Deutschland: Fachschule, Fachhochschul- oder Hochschulabschluss). Desweiteren werden auf Basis dreier binärer Variablen verschiedene Dimensionen von Gesundheit unterschieden: die subjektive Einschätzung des Gesundheitszustandes durch die Befragten (,gut oder besser' vs. ,mittelmäßig oder schlechter'), das Vorhandensein chronischer Krankheiten (,2 oder mehr' vs. ,1 oder keine'), sowie das Auftreten von Depressionssymptomen in den vier dem Interview vorangegangenen Wochen (auf Basis der so genannten Euro-D-Skala). Schließlich berücksichtigen wir die mögliche Wechselwirkung zwischen Aktivitäten in verschiedenen Bereichen informeller Arbeit durch die Aufnahme von Ehrenamt, Netzwerkhilfe bzw. Pflege (je nach Modell) als binäre Kontrollvariablen auf der rechten Seite der Regressionsgleichung.

Unsere zentralen erklärenden (Makro-)Variablen sind die Erwerbsbeteiligung Älterer, der Freedomhouse Index of Civil Liberties und der Anteil der Sozialausgaben am Bruttoinlandsprodukt (BIP). Die Erwerbsbeteiligung Älterer wird als die von Eurostat für das Jahr 2004 ausgewiesene Erwerbsquote der 55- bis 64-Jährigen gemessen (vgl. http://epp.eurostat.ec.europa.eu/). Ebenfalls auf Angaben von Eurostat beruhen die Angaben zum prozentualen Anteil der Sozialausgaben am BIP im Jahr 2004. Berücksichtigt werden monetäre Leistungen des Staates an private Haushalte in den folgenden Bereichen: Krankheit, Invalidität, Gebrechen, Arbeitsunfall, Berufskrankheit, Alter, Hinterbliebene, Mutterschaft, Familie, Beschäftigungsförderung, Arbeitslosigkeit, Wohnung, Ausbildung und allgemeiner Bedürftigkeit. Als Indikator für den gesellschaftlichen Grad an religiöser und politischer Freiheit dient schließlich der Freedomhouse Index of Civil Liberties (vgl. http://www.freedomhouse.org), der sich wie die anderen Makroindikatoren auf das Jahr 2004 bezieht. Dieser Index wird auf der Basis von Experteneinschätzungen erstellt und setzt sich aus vier Unterkategorien zusammen: Meinungs- und Religionsfreiheit, 
Vereinigungsfreiheit und Organisationsrechte, Rechtsstaatlichkeit, persönliche Autonomie und individuelle Rechte. Dabei werden nicht nur formalrechtliche Regelungen berücksichtigt, sondern es wird auch die aktuelle Praxis in die Bewertung einbezogen. Der Wertebereich des Indexes liegt zwischen 0 und 60 Punkten.

\subsection{Methodisches Vorgehen}

Für die multivariate Analyse bedienen wir uns des Instrumentariums der Mehrebenenanalyse und schätzen hier so genannte random intercept Modelle für binäre abhängige Variablen (vgl. Guo \& Zhao 2000; Snijders \& Bosker 1999: Kapitel 4). Bei diesem Verfahren kann die Regressionskonstante über die Länder hinweg variieren, d.h. sie besteht aus einer fixen Komponente und einem Zufallskoeffizienten in Form eines normalverteilten Fehlerterms. Dieser nimmt für alle Beobachtungen innerhalb eines Landes den gleichen Wert an und beschreibt die Abweichung jedes einzelnen Kontexts vom fixen Teil der Konstante (between-context variance). Damit wird nicht nur die Korrelation zwischen Individuen innerhalb desselben Landes statistisch kontrolliert, sondern es werden auch sonst unbeobachtete Kontexteffekte erfasst. Unterscheidet sich die Standardabweichung des regionalen Fehlerterms - in den Tabellen als $\sigma_{u}$ ausgewiesen - statistisch signifikant von 0, liegen solche Effekte vor. - Die Ergebnisse der logistischen Regressionen werden als Odds Ratios präsentiert.

\section{Empirische Ergebnisse}

\subsection{Deskriptive Befunde}

Vor der multivariaten Mehrebenenanalyse sollen die Abbildungen 1-3 zunächst einen knappen deskriptiven Eindruck von der Beteiligung Älterer an den hier betrachteten 
Dimensionen informeller Arbeit im Ländervergleich geben ${ }^{2}$ und den bivariaten Zusammenhang zwischen den jeweiligen Engagementquoten und unseren drei Makroindikatoren illustrieren. Die einzelnen Punktdiagramme sind dabei in Quadranten unterteilt, die durch die Mittelwerte (Median) der X- und Y-Variablen definiert sind.

Im Monat vor der Befragung engagierten sich durchschnittlich etwa 20 Prozent der über 50-Jährigen in der Netzwerkhilfe, ca. 10 Prozent übten ein Ehrenamt aus und 5 Prozent pflegten einen anderen Erwachsenen. Insbesondere in den Bereichen Netzwerkhilfe und Ehrenamt zeichnet sich dabei ein deutliches regionales Muster mit hohen Engagementquoten in Belgien, Dänemark, den Niederlanden und Schweden, sowie vergleichsweise niedrigen Anteilen aktiver Älterer in Italien, Spanien und Griechenland ab. Mit 15-20 Prozent ehrenamtlich Aktiven und einem Drittel Netzwerkhilfe Leistender sind die Anteile der in diesen beiden Bereichen engagierten ,Nord'-Europäer (einschließlich Belgier und Niederländer) bis zu doppelt so hoch wie im europäischen Durchschnitt, während - neben den Italienern und Griechen insbesondere die Spanier deutlich unterdurchschnittliche Werte aufweisen (Ehrenamt: 2 Prozent; Netzwerkhilfe: 6 Prozent). Länderunterschiede - in der Spanne von 9 Prozent in Belgien bis 2 Prozent in Italien und Spanien - finden sich auch bei informellen Pflegeleistungen, allerdings ist hier ein insgesamt etwas weniger eindeutiges räumliches Muster (im Sinne eines klaren Nord-Süd-Gradienten) zu erkennen.

Hinsichtlich des bivariaten Zusammenhangs zwischen der Beteiligung an informellen produktiven Tätigkeiten - aggregiert auf der Länderebene - und den Makroindikatoren Erwerbsquote der 55- bis 64-Jährigen, Civil Liberties Index und Anteil der Sozialausgaben zeigt sich jeweils ein Muster, dass zumindest in der Tendenz den von uns formulierten Hypothesen entspricht. Höhere Engagementquoten (auf der X-Achse) gehen im Allgemeinen mit höheren Werten der Makroindikatoren (auf der Y-

\footnotetext{
2 Detaillierte deskriptive Analysen, einschließlich einer weiteren Differenzierung nach verschiedenen soziodemographischen und sozioökonomischen Merkmalen, finden sich bei Erlinghagen \& Hank 2006; Hank \& Stuck 2007; Stuck 2006.
} 
Achse) einher, d.h. die Ländergruppe Belgien, Dänemark, Niederlande und Schweden findet sich meist relativ geschlossen im Quadranten I der Punktdiagramme, während die Mittelmeerländer im Quadranten III konzentriert sind. Ausnahmen von diesem Muster sind im Wesentlichen auf die geringe Erwerbsbeteiligung Älterer in Belgien und den niedrigen Anteil Pflegender in Dänemark zurückzuführen. Die Verteilung der Länder mit mittlerer Partizipation (Deutschland, Frankreich, Österreich und die Schweiz) erweist sich als wenig systematisch.

[Abbildungen 1-3 etwa hier einfügen]

\subsection{Multivariate Ergebnisse}

Die Ergebnisse der multivariaten Analysen für die drei abhängigen Variablen ,Ehrenamt' (Tabelle 2), ,Netzwerkhilfe' (Tabelle 3) und ,Pflege' (Tabelle 4) werden gemeinsam präsentiert. Die endgültigen Regressionsmodelle werden ausgehend von einem ,leeren' Modell, das nur die Konstante und den regionalen Zufallseffekt enthält (Modell 1), schrittweise aufgebaut. Die individuellen Kontrollvariablen werden jeweils in Modell 2 eingeführt; die Makroindikatoren folgen in Modell 3.

Die ,leeren' Modelle zeigen deutlich die signifikante regionale Variation der Konstante. Der Anteil der Varianz zwischen den Ländern (also der ,between-context variance') an der Gesamtvarianz - im Folgenden mit $\rho$ bezeichnet - reicht von 14 Prozent im Modell für das ehrenamtliche Engagement und 10 Prozent bei der Netzwerkhilfe bis zu lediglich 3 Prozent im Modell für die Pflege. Die Aufnahme der individuellen Kontrollvariablen (Modell 2) reduziert die jeweiligen Varianzkomponenten auf 10 Prozent, 8 Prozent bzw. etwas mehr als 1 Prozent. Dies bedeutet, dass zwischen einem Fünftel (für die Netzwerkhilfe) und mehr als der Hälfte (bei der Pflege) der Varianz zwischen den Ländern auf deren unterschiedliche Bevölkerungskomposition, so wie sie durch die in den Regressionsmodellen 
berücksichtigten Individualvariablen abgebildet wird, zurückgeführt werden kann. Dennoch bleiben alle $\rho$ 's statistisch hochsignifikant.

Für alle drei abhängigen Variablen belegen die Koeffizienten der individuellen Kontrollvariablen eine gleichermaßen große Bedeutung individueller Ressourcen für die Ausübung produktiver Tätigkeiten (siehe auch Hank \& Stuck 2007). Hinsichtlich der Wahrscheinlichkeit, informelle Arbeit zu leisten, zeigt sich im Allgemeinen ein negativer Altersgradient. Während die Variable, Geschlecht' keinen statistisch signifikanten Beitrag zur Erklärung von Unterschieden ehrenamtlichen Engagements leistet, weisen Frauen eine höhere Wahrscheinlichkeit auf, informelle Hilfe bzw. insbesondere Pflege zu leisten. Der Partnerschaftsstatus weist mit keiner der abhängigen Variablen einen statistisch signifikanten Zusammenhang auf. Ein positiver Bildungsgradient zeigt sich unabhängig von der konkreten Tätigkeit, jedoch besonders deutlich im Bereich des ehrenamtlichen Engagements. Auch der - nun allerdings negative - Zusammenhang zwischen informeller Arbeit und Erwerbsarbeit (vs. Ruhestand) ist hier stärker als in der Schätzung für ,Netzwerkhilfe', und für die ,Pflege’ ist der entsprechende Koeffizient sogar insignifikant. Interessant ist, dass auch für Nicht-Erwerbstätige die Wahrscheinlichkeit informelle Hilfe zu leisten geringer ausfällt als für Rentner - ein möglicher Hinweis darauf, dass hier Netzwerke aus der Erwerbsphase eine nachhaltige Rolle spielen könnten. Ein relativ uneinheitliches Bild ergibt sich hinsichtlich der Beziehung von informeller Arbeit mit verschiedenen Dimensionen der Gesundheit. Die Wahrscheinlichkeit ein Ehrenamt auszuüben reduziert sich signifikant, wenn der eigene Gesundheitszustand als mittelmäßig oder schlecht wahrgenommen wird bzw. wenn Symptome von Depressionen vorliegen. Ein negativer Zusammenhang kann auch zwischen subjektiver Gesundheit und informeller Hilfe festgestellt werden. Bei der Netzwerkhilfe weisen jedoch Befragte mit zwei oder mehr chronischen Krankheiten, die unter Umständen selbst hilfebedürftig sind, eine höhere Wahrscheinlichkeit auf, aktiv zu sein. Dies könnte als Hinweis darauf 
interpretiert werden, dass in diesem Bereich Reziprozität eine Rolle spielt. Bei der Pflege fällt vor allem der hochsignifikante Koeffizient der Depressions-Variable auf, dessen Richtung auf eine höhere Prävalenz seelischer Probleme unter Pflegenden anzeigt. In allen Schätzungen findet sich eine stark positive, hochsignifikante Korrelation zwischen der abhängigen Variable und den jeweils anderen produktiven Tätigkeiten, d.h. auch wenn andere individuelle Merkmale in der statistischen Analyse berücksichtigt werden ergeben sich Hinweise darauf, dass die Wahrscheinlichkeit in einem Bereich aktiv zu sein mit parallel hierzu stattfindenden Aktivitäten in anderen Bereichen steigt (vgl. zu diesem Aspekt ausführlich Hank \& Stuck 2007).

Die Koeffizienten der Kontrollvariablen bleiben nach Einführung der Makroindikatoren in Modell 3 substantiell unverändert. Die Erwerbsquote der 55- bis 64-Jährigen weist mir keiner der abhängigen Variablen einen statistisch signifikanten Zusammenhang auf. Der Index of Civil Liberties ist positiv signifikant mit der Wahrscheinlichkeit ein Ehrenamt auszuüben korreliert und erweist sich auch in der Regression für die Netzwerkhilfe als (schwach) signifikant. Ein durchgängig positiver Koeffizient - für das Ehrenamt allerdings nur auf dem 10-Prozent-Niveau statistisch signifikant - findet sich für den Anteil der Sozialausgaben, d.h. die individuelle Neigung, im Alter einer informell-produktiven Tätigkeit nachzugehen (insbesondere im Bereich Netzwerkhilfe), hängt auch vom Engagement des Wohlfahrtsstaates ab. Obwohl der Anteil der ,between-context variance' in Modell 3 nochmals deutlich sinkt - auf maximal 3 Prozent in der Schätzung des ehrenamtlichen Engagements - bleibt für alle hier berücksichtigten Dimensionen produktiven Alterns ein statistisch hochsignifikanter Rest unbeobachteter regionaler Heterogenität erhalten.

[Tabellen 2-4 etwa hier einfügen] 


\section{Schlussfolgerungen}

Hauptanliegen des vorliegenden Beitrages war es, zu einem besseren Verständnis der gesellschaftlichen Determinanten der unterschiedlich starken Beteiligung älterer Europäer an informellen produktiven Tätigkeiten beizutragen. Auf Basis von Daten des Survey of Health, Ageing and Retirement in Europe und unter Verwendung logistischer Mehrebenenmodelle konnten wir zeigen, dass ein substantieller Teil der Varianz zwischen den elf hier betrachteten Ländern hinsichtlich des privaten Engagements in den Bereichen Ehrenamt, Netzwerkhilfe und Pflege auf Unterschiede in der Bevölkerungskomposition (z.B. die Verteilung von Bildungsabschlüssen) zurück geführt werden kann. Darüber hinaus tragen auch die drei von uns untersuchten Makroindikatoren erheblich zur Erklärung des Varianzanteils auf der Länderebene bei. Während gesellschaftliche Altersbilder - so wie sie in dieser Studie operationalisiert wurden - jedoch keinen signifikanten Zusammenhang mit ehrenamtlichem Engagement, Netzwerkhilfe und Pflege aufweisen, konnten unsere Hypothesen zur Bedeutung von politischer und religiöser Freiheit sowie wohlfahrtsstaatlichem Engagement weitgehend bestätigt werden, d.h.: je positiver ein Land im Freedomhouse Civil Liberties Index abschneidet bzw. je größer der Anteil der Sozialausgaben am BIP, desto stärker neigen ältere Bürger dazu, selbst produktiv tätig zu sein, insbesondere in den Bereichen Ehrenamt und Netzwerkhilfe.

Aus den hier vorgestellten empirischen Befunden lassen sich unseres Erachtens im Wesentlichen zwei sozialpolitisch und sozialwissenschaftlich relevante Schlussfolgerungen ziehen (vgl. auch Hank et al. 2006: 10f.). Es ist, erstens, festzustellen, dass die europäischen Wohlfahrtsstaaten die Verantwortung für die Ausweitung privaten (,bürgerschaftlichen') Engagements in der älteren Bevölkerung und damit eine Stärkung des produktiven Alter(n)s - nicht den Bürgern und Bürgerinnen allein überlassen dürfen bzw. können, sondern dass es hier unterstützender staatlicher Maßnahmen bedarf (vor allem in jenen Ländern, in denen es bislang nur eine 
verhältnismäßig schwach ausgeprägte ,Kultur’ informeller Arbeit gibt). D.h., wenn Aufgaben, die prinzipiell auch der Wohlfahrtsstaat übernehmen könnte, auf die ,Zivilgesellschaft' übertragen werden sollen, muss sich die Politik darüber im Klaren sein, dass dies nicht ohne öffentliche Investitionen in hierfür notwendige Rahmenbedingungen funktionieren wird. Entsprechende wohlfahrtsstaatliche Interventionspunkte dürften vor allem auf lokaler Ebene von konkreter Bedeutung sein, in dem etwa gemeinnützige Einrichtungen, Nachbarschaftsinitiativen oder Programme gefördert werden, die gezielt auf die Aktivierung und Einbindung von Senioren in informelle produktive Tätigkeiten gerichtet sind (vgl. Brauers 2007; Breithecker 2007). Neben einem gesellschaftlichen Nutzen, der aus der Aktivierung bislang ungenutzter produktiver Potenziale Älterer resultieren würde, dürfte dies auch zur Verbesserung der Lebensqualität der Aktiven selbst beitragen - solange eine angemessene Anerkennung des Geleisteten erfolgt (z.B. Wahrendorf \& Siegrist 2007)! Es muss nämlich Sorge dafür getragen werden, dass neben den Möglichkeiten informeller Arbeit in einem produktiven Alter auch dessen Grenzen berücksichtigt und überzogene Erwartungen an die älter werdende Zivilgesellschaft vermieden werden (z.B. Aner \& Hammerschmidt 2007; Erlinghagen 2007b).

Hier kann, zweitens, weitere sozialwissenschaftliche Forschung einen wichtigen Beitrag leisten, in dem sie Hinweise darauf liefert, unter welchen gesellschaftlichen Bedingungen und in welchem Ausmaß das produktive Potenzial Älterer nachhaltig aktiviert werden kann. Eine besondere Bedeutung spielt hier neben dem Wohlfahrtsstaat die Familie. Pichler \& Wallace (2007) zeigen, dass etwa in den südeuropäischen Ländern starke Bindungen innerhalb des Familienverbandes zu einer relativ schwachen Ausprägung sozialer Netzwerke jenseits der Familie führen. D.h., ein stärkeres Engagement des mediterranen Wohlfahrtsstaates zur Förderung informeller Arbeit und produktiven Alterns ohne Berücksichtigung der Rolle familiärer Unterstützungsnetzwerke würde womöglich ins Leere laufen. Die Funktionsweise der komplexen Interaktionen innerhalb der Triade ,Wohlfahrtsstaat - Familie - soziale 
Netzwerke' lässt sich mutmaßlich nur kontextspezifisch verstehen: eine einheitliche europäische Aktivierungsstrategie scheint daher nur bedingt Erfolg versprechend. Zielgerichtete Programme zur Stärkung des produktiven Engagements im Alter sollten auch nationale Eigenheiten hinsichtlich der jeweils dominierenden Tätigkeitsbereiche, in denen informelle Arbeit ausgeübt wird, berücksichtigen (vgl. Curtis et al. 2001: 786f.; Salamon \& Sokolowski 2003: 75f.). Nur wenn wir besser als bislang verstehen, welche Bedeutung etwa der Begriff des ,Ehrenamtes' in verschiedenen Ländern hat (z.B. Anheier \& Toepler 2002: 37f.) und welche Tätigkeitsinhalte sich in erster Linie damit verbinden, können konstruktive und konkrete Aussagen darüber gemacht werden, welche - jeweils kontextspezifischen - Fördermaßnahmen ergriffen werden sollten, und welche Wirkung von diesen erwartet werden kann.

\section{Danksagung}

Wir bedanken uns bei Marcel Erlinghagen für konstruktive Kommentare sowie bei der Fritz Thyssen Stiftung für die finanzielle Förderung des Projektes „Informelle Arbeit von Älteren in Deutschland und Europa“, in dessen Rahmen der vorliegende Beitrag entstanden ist. Die SHARE-Datenerhebung 2004 wurde hauptsächlich durch das 5. Rahmenprogramm der Europäischen Union finanziert (Projekt QLK6-CT-2001-00360). Weitere Finanzmittel wurden vom U.S. National Institute on Aging zur Verfügung gestellt (U01 AG09740-13S2, P01 AG005842, P01 AG08291, P30 AG12815, Y1-AG4553-01 and OGHA 04-064). Die Datensammlung in Belgien (durch das Belgian

Science Policy Office), Österreich (durch den Fonds zur Förderung der wissenschaftlichen Forschung) und der Schweiz (durch BBW/OFES/UFES) wurde national finanziert. Darüber hinaus danken wir der Europäischen Union für Unterstützung durch das 6. Rahmenprogramm (Projekt RII-CT-2006-026193; SHAREI3). 


\section{Literatur}

Aner, K. / Hammerschmidt, P. (2007): Zivilgesellschaftlich produktiv altern. Eine kritische Analyse ausgewählter Modellprogramme, in: M. Erlinghagen \& K. Hank (Hrsg.), Produktives Altern und informelle Arbeit in modernen Gesellschaften. VS Verlag: Wiesbaden, im Druck.

Anheier, H.K. / Toepler, S. (2002): Bürgerschaftliches Engagement in Europa. Aus Politik und Zeitgeschichte B 9/2002, 31-38.

Bellmann, L. / Kistler, E. / Wahse, J. (2003): Betriebliche Sicht- und Verhaltensweisen gegenüber älteren Arbeitnehmern. Aus Politik und Zeitgeschichte B 20/2003, 2634.

Börsch-Supan, A. / Hank, K. / Jürges, H. (2005): A new comprehensive and international view on ageing: Introducing the 'Survey of Health, Ageing and Retirement in Europe'. European Journal of Ageing 2, 245-253.

Börsch-Supan, A. / Jürges, H. (Hrsg.) (2005): The Survey of Health, Ageing and Retirement in Europe - Methodology. MEA: Mannheim.

Brauers, S. (2007): Potenziale der Älteren in Kommunen nutzen. Das Bundesmodellprogramm „Erfahrungswissen für Initiativen“ im europäischen Kontext, in: M. Erlinghagen \& K. Hank (Hrsg.), Produktives Altern und informelle Arbeit in modernen Gesellschaften. VS Verlag: Wiesbaden, im Druck.

Breithecker, R. (2007): Potenziale bürgerschaftlichen Engagements für die Kommune. Das Modellprojekt „Selbstorganisation älterer Menschen“, in: M. Erlinghagen \& K. Hank (Hrsg.), Produktives Altern und informelle Arbeit in modernen Gesellschaften. VS Verlag: Wiesbaden, im Druck.

Brugiavini, A. / Croda, E. / Mariuzzo, F. (2005): Labour force participation of the elderly: Unused capacity?, in: A. Börsch-Supan et al. (Hrsg.), Health, Ageing and Retirement in Europe - First Results from the Survey of Health, Ageing and Retirement in Europe. MEA: Mannheim, 236-240. 
Caro, F.G. (2007): Produktives Altern und ehrenamtliches Engagement in den USA, in: M. Erlinghagen \& K. Hank (Hrsg.), Produktives Altern und informelle Arbeit in modernen Gesellschaften. VS Verlag: Wiesbaden, im Druck.

Curtis, J.E. / Bear, D.E. / Grabb, E.G. (2001): Nations of Joiners: Explaining Voluntary Association Membership in Democratic Societies. American Sociological Review 66, 783-805.

Erlinghagen, M. (2000): Informelle Arbeit. Ein Überblick über einen schillernden Begriff. Schmollers Jahrbuch 120, 239-274.

Erlinghagen, M. (2007a): Ehrenamtliche Arbeit und informelle Hilfe nach dem Renteneintritt, in: M. Erlinghagen \& K. Hank (Hrsg.), Produktives Altern und informelle Arbeit in modernen Gesellschaften. VS Verlag: Wiesbaden, im Druck.

Erlinghagen, M. (2007b): Informelle Arbeit in alternden Gesellschaften. Möglichkeiten und Grenzen des ,produktiven Alterns'. Unveröffentlichtes Manuskript, RuhrUniversität Bochum.

Erlinghagen, M. / Hank, K. (2006): The Participation of Older Europeans in Volunteer Work. Ageing \& Society 26, 567-584.

Erlinghagen, M. / Hank, K. (Hrsg.) (2007): Produktives Altern und informelle Arbeit in modernen Gesellschaften. VS Verlag: Wiesbaden, im Druck.

Esping-Andersen, G. (1990): The Three Worlds of Welfare Capitalism. Princeton University Press: Princeton.

Filipp, S.-H. / Mayer, A.-K. (2005): Zur Bedeutung von Altersstereotypen. Aus Politik und Zeitgeschichte B 49-50/2005, 25-31.

Göckenjan, G. (2007): Zur Wandlung des Altersbildes seit den 1950er Jahren im Kontext und als Folge der Großen Rentenreform von 1957. Deutsche Rentenversicherung 62, 125-142.

Goss, K. (1999): Volunteering and the Long Civic Generation. Nonprofit and Voluntary Sector Quarterly 28, 378-415.

Gruber, J. / Wise, D. (Hrsg.) (2004): Social Security Programs and Retirement around the World: Micro-Estimation. University of Chicago Press: Chicago. 
Guo, G. / Zhao, H. (2000): Multilevel Modeling for Binary Data. Annual Review of Sociology 26, 441-462.

Hank, K. / Buber, I. (2007): Grandparents Caring for Their Grandchildren: Findings from the 2004 Survey of Health, Ageing and Retirement in Europe. MEA Discussion Paper 127-07, Universität Mannheim.

Hank, K. / Erlinghagen, M. / Lemke, A. (2006): Ehrenamtliches Engagement in Europa: Eine Vergleichende Untersuchung am Beispiel von Senioren. Sozialer Fortschritt $55,6-12$.

Hank, K. / Stuck, S. (2007): Ehrenamt, Netzwerkhilfe und Pflege in Europa Komplementäre oder konkurrierende Dimensionen produktiven Alterns?, in: M. Erlinghagen \& K. Hank (Hrsg.), Produktives Altern und informelle Arbeit in modernen Gesellschaften. VS Verlag: Wiesbaden, im Druck.

Kääriäinen, J. / Lehtonen, H. (2006): The variety of social capital in welfare state regimes - A comparative study of 21 countries. European Societies 8, 27-57.

Knuth, M. / Kalina, T. (2002): Early exit from the labour force between exclusion and privilege: Unemployment as a transition from employment to retirement in West Germany. European Societies 4, 393-418.

Kohli, M. / Rein, M. / Guillemard, A.-M. / van Gunsteren, H. (Hrsg.) (1991): Time for Retirement: Comparative Studies of Early Exit from the Labor Force. Cambridge University Press: Cambridge.

Kruse, A. / Schmitt, E. (2005): Zur Veränderung des Altersbildes in Deutschland. Aus Politik und Zeitgeschichte B 49-50/2005, 9-17.

Künemund, H. (2005): „Produktive“ Tätigkeiten, in: M. Kohli \& H. Künemund (Hrsg.), Die zweite Lebenshälfte (2., erw. Auflage). VS Verlag: Wiesbaden, 277-317.

Motel-Klingebiel, A. / Tesch-Römer, C. / von Kondratowitz, J. (2005): Welfare states do not crowd out the family: evidence for mixed responsibility from comparative analyses. Ageing \& Society 25, $863-882$.

Morrow-Howell, N. / Hinterlong, J./ Sherraden, M. (Hrsg.) (2001): Productive Aging: Concepts and Challenges. Johns Hopkins University Press: Baltimore. 
Mutchler, J.E. / Burr, J.A. / Caro, F.G. (2003): From Paid Worker to Volunteer: Leaving the Paid Labor Force and Volunteering in Later Life. Social Forces 81, 12671293.

Pfau-Effinger, B. (2005): Culture and Welfare State Policies: Reflections on a Complex Interaction. Journal of Social Policy 34, 3-20.

Pichler, F. / Wallace, C. (2007): Patterns of Formal and Informal Social Capital in Europe. European Sociological Review 23, 423-435.

Roscigno, V.J. / Mong, S. / Byron, R. / Tester, G. (2007): Age Discrimination, Social Closure and Employment. Social Forces 86, 313-334.

Rotolo, T. / Wilson, J. (2004): What Happens to the "Long Civic Generation"? Explaining Cohort Differences in Volunteerism. Social Forces 82, 1091-1121.

Salamon, L.M. / Sokolowski, S.W. (2003): Institutional Roots of Volunteering: Toward a Macro-Strucutral Theory of Individual Voluntary Action, in: P. Dekker \& L. Halman (Hrsg.), The Values of Volunteering. Cross-Cultural Perspectives. Kluwer Academic / Plenum Publishers: New York u.a., 71-90.

Salamon, L.M. / Sokolowski, S.W. / List, R. (2003): Global Civil Society. An Overview. Johns Hopkins University: Baltimore.

Schmid, J. (2002): Wohlfahrtsstaaten im Vergleich. Soziale Sicherung in Europa: Organisation, Finanzierung, Leistungen und Probleme (2. völlig überarbeitete und erweiterte Auflage). Leske und Budrich: Opladen.

Smith, D.H. / Shen, C. (2002): The Roots of Civil Society: A Model of Voluntary Association Prevalence Applied to Data on Larger Contemporary Nations. International Journal of Comparative Sociology 43, 93-133.

Snijders, T.A.B. / Bosker, R.J. (1999): Multilevel Analysis: An Introduction to Basic and Advanced Multilevel Modeling. Sage: London.

Staudinger, U.M. (2003): Das Alter(n): Gestalterische Verantwortung für den Einzelnen und die Gesellschaft. Aus Politik und Zeitgeschichte B 20/2003, 35-42.

Stuck, S. (2006): Informelle Arbeit älterer Frauen und Männer in Europa. Zeitschrift für Frauenforschung \& Geschlechterstudien 24, 117-130. 
Tang, F. (2006): What resources are needed for volunteerism? A life course perspective. Journal of Applied Gerontology 25, 375-390.

van Oorschot, W. / Arts, W. (2005): The social capital of European welfare states: the crowding out hypothesis revisited. Journal of European Social Policy 15, 5-26.

Wahrendorf, M. / Siegrist, J. (2007): Soziale Produktivität und Wohlbefinden im höheren Lebensalter, in: M. Erlinghagen \& K. Hank (Hrsg.), Produktives Altern und informelle Arbeit in modernen Gesellschaften. VS Verlag: Wiesbaden, im Druck.

Wilson, J. / Musick, M. (1998): The Contribution of Social Resources to Volunteering. Social Science Quarterly 79, 799-814.

\section{Abbildungen \& Tabellen}


Abbildung 1: Zusammenhang zwischen dem Anteil ehrenamtlich Aktiver über 50-

\section{Jähriger und Makroindikatoren in 11 Ländern}
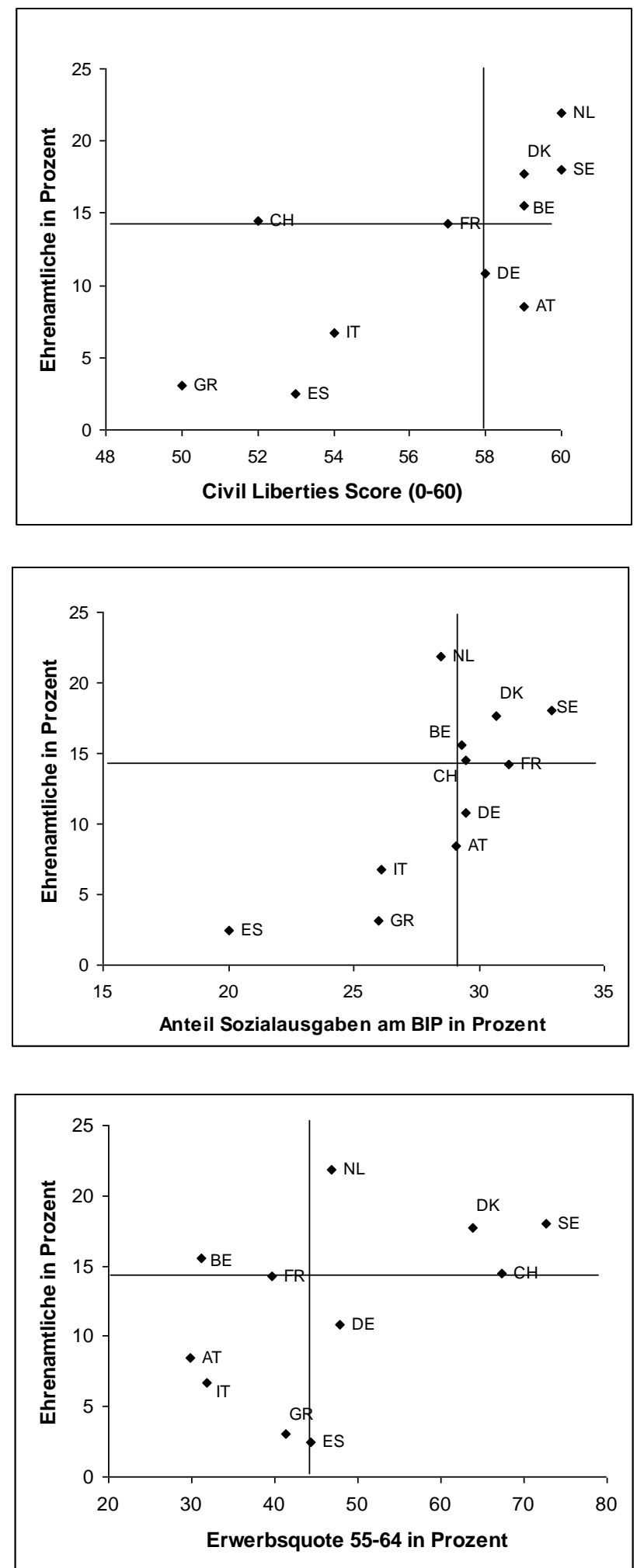

Quelle: SHARE 2004 (Release 2.0.1; gewichtet), Eurostat (2004), Freedomhouse (2004). 
Abbildung 2: Zusammenhang zwischen dem Anteil in der Netzwerkhilfe Aktiver über 50-Jähriger und Makroindikatoren in 11 Ländern
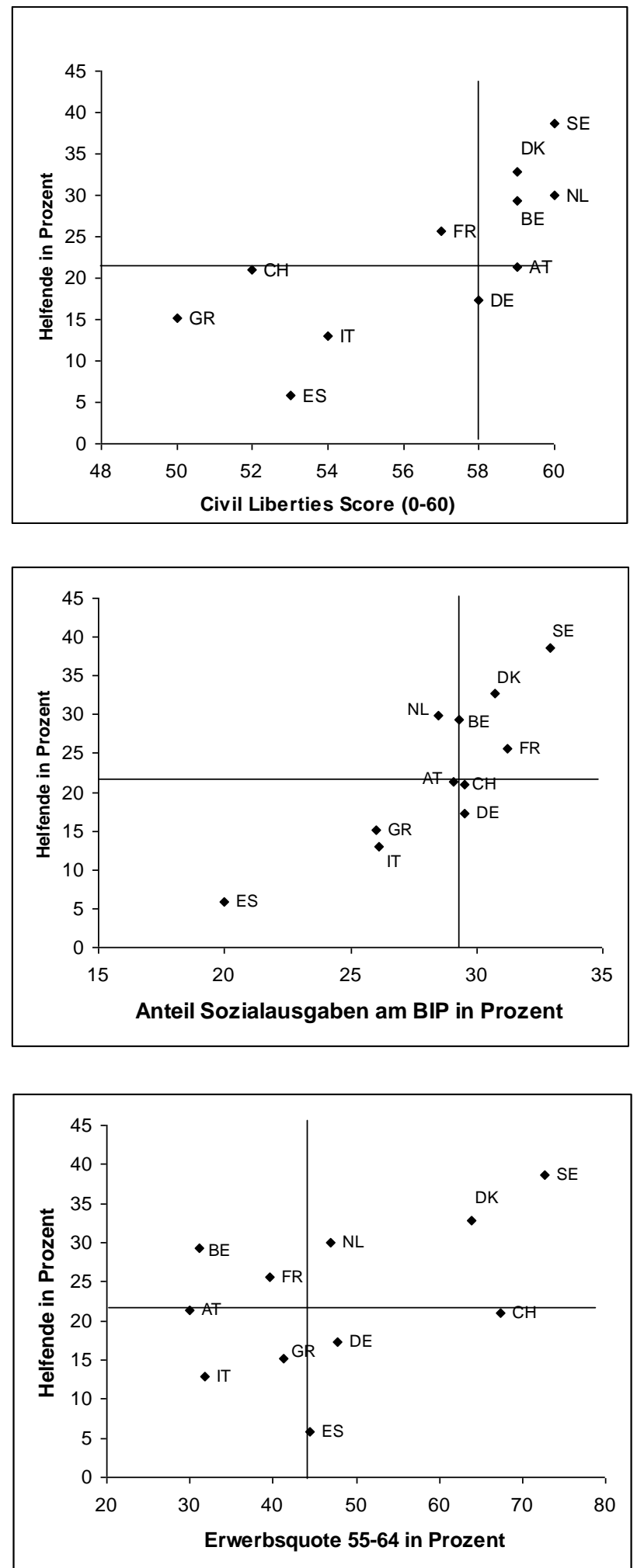

Quelle: SHARE 2004 (Release 2.0.1; gewichtet), Eurostat (2004), Freedomhouse (2004). 
Abbildung 3: Zusammenhang zwischen dem Anteil Pflegender über 50-Jähriger und Makroindikatoren in 11 Ländern
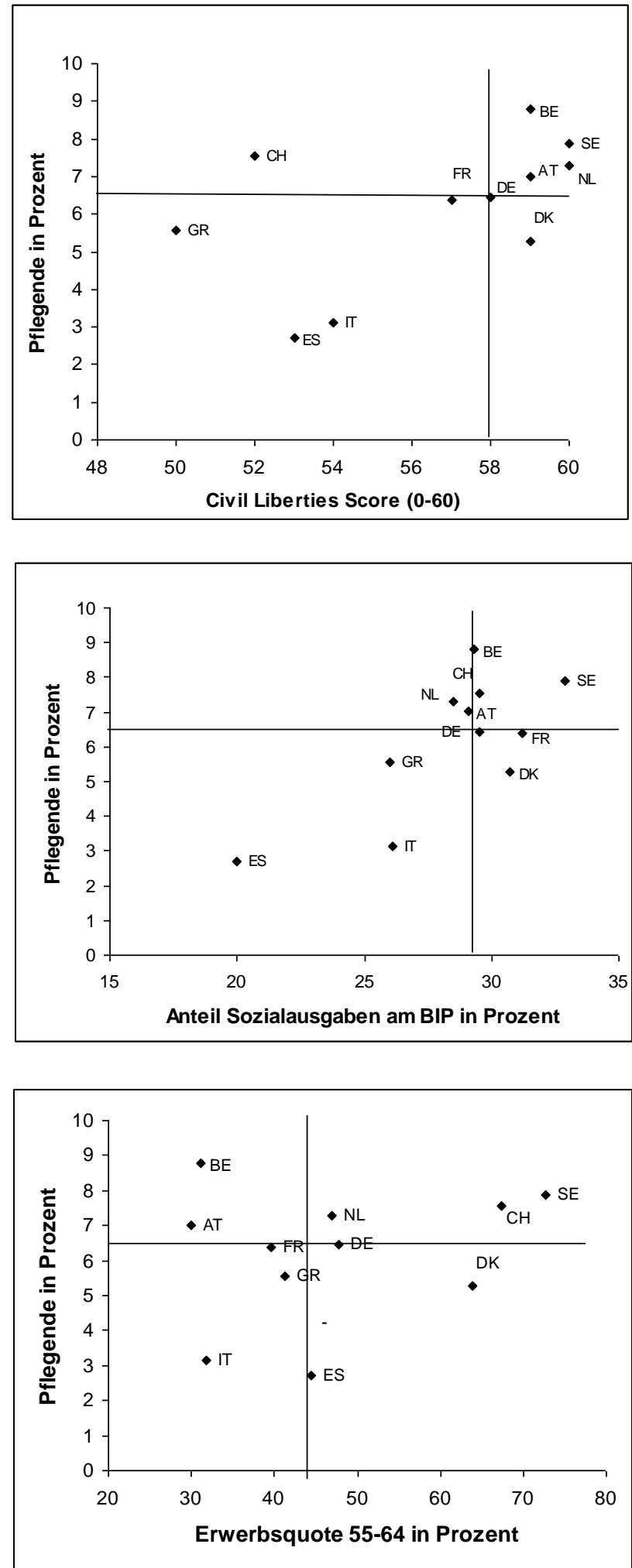

Quelle: SHARE 2004 (Release 2.0.1; gewichtet), Eurostat (2004), Freedomhouse (2004). 
Tabelle 1: Deskriptive Statistiken der Stichprobe (ungewichtet)

\begin{tabular}{|c|c|}
\hline & Anteile in Prozent \\
\hline \multicolumn{2}{|l|}{ Dimensionen informeller Arbeit } \\
\hline Aktiv im Bereich ,Ehrenamt' & 12 \\
\hline Aktiv im Bereich ,Netzwerkhilfe' & 23 \\
\hline Aktiv im Bereich ,Pflege' & 6 \\
\hline \multicolumn{2}{|l|}{ Demographische Merkmale } \\
\hline Geschlecht weiblich & 54 \\
\hline Alter 50-64 & 53 \\
\hline Alter $65-74$ & 27 \\
\hline Alter 75+ & 19 \\
\hline Mit Partner lebend & \\
\hline \multicolumn{2}{|l|}{ Sozio-ökonomische Merkmale } \\
\hline Erwerbstätig & 28 \\
\hline Nicht erwerbstätig & 23 \\
\hline Im Ruhestand & 50 \\
\hline Niedriger Bildungsabschluss & 52 \\
\hline Mittlerer Bildungsabschluss & 29 \\
\hline Hoher Bildungsabschluss & 19 \\
\hline \multicolumn{2}{|l|}{ Gesundheitsmerkmale } \\
\hline Subjektive Gesundheit: mittelmäßig oder schlechter & 38 \\
\hline $2+$ chronische Krankheiten & 41 \\
\hline Depressionssymptome & 24 \\
\hline$(\mathrm{N}=27.266)^{\mathrm{a}}$ & \\
\hline
\end{tabular}

${ }^{\text {a }}$ Belgien: 3.684; Dänemark: 1.606; Deutschland: 2.934; Frankreich: 2.943; Griechenland: 2.676; Italien: 2.493; Niederlande: 2.851; Österreich: 1.839; Schweden: 2.994; Spanien: 2.329; Schweiz: 954.

Quelle: SHARE 2004 (Release 2.0.1), eigene Berechnungen. 
Tabelle 2: Ergebnisse der logistischen Mehrebenenmodelle für die abhängige Variable ,Ehrenamt'

\begin{tabular}{|c|c|c|c|c|c|c|}
\hline \multirow[b]{2}{*}{ Variablen } & \multicolumn{2}{|c|}{ Modell 1} & \multicolumn{2}{|c|}{ Modell 2} & \multicolumn{2}{|c|}{ Modell 3} \\
\hline & OR & s.e. & OR & s.e. & OR & s.e. \\
\hline \multicolumn{7}{|l|}{ Demographische Merkmale } \\
\hline Geschlecht weiblich & & & 0,93 & 0,03 & 0,93 & 0,03 \\
\hline Alter $50-64^{\mathrm{a}}$ & & & & & & \\
\hline Alter $65-74$ & & & 0,95 & 0,05 & 0,95 & 0,05 \\
\hline Alter $75+$ & & & $0,55 * *$ & 0,04 & $0,55 * *$ & 0,04 \\
\hline Mit Partner lebend & & & 1,02 & 0,04 & 1,02 & 0,04 \\
\hline \multicolumn{7}{|l|}{ Sozio-ökonomische Merkmale } \\
\hline Erwerbstätig & & & $0,64 * *$ & 0,03 & $0,64 * *$ & 0,03 \\
\hline Nicht erwerbstätig & & & 0,90 & 0,05 & 0,91 & 0,05 \\
\hline Im Ruhestand ${ }^{\mathrm{a}}$ & & & & & & \\
\hline Niedriger Bildungsabschluss ${ }^{\mathrm{a}}$ & & & & & & \\
\hline Mittlerer Bildungsabschluss & & & $1,38 * *$ & 0,06 & $1,37 * *$ & 0,06 \\
\hline Hoher Bildungsabschluss & & & $2,26 * *$ & 0,11 & $2,25 * *$ & 0,11 \\
\hline \multicolumn{7}{|l|}{ Gesundheitsmerkmale } \\
\hline Subjektive Gesundheit & & & $0,72 * *$ & 0,03 & $0,72 * *$ & 0,03 \\
\hline $2+$ chronische Krankheiten & & & 1,05 & 0,04 & 1,05 & 0,04 \\
\hline Depressionssymptome & & & $0,79 * *$ & 0,04 & $0,79 * *$ & 0,04 \\
\hline \multicolumn{7}{|l|}{ Dimensionen informeller Arbeit } \\
\hline Netzwerkhilfe & & & $1,79 * *$ & 0,07 & $1,79 * *$ & 0,07 \\
\hline Pflege & & & $2,37 * *$ & 0,14 & $2,36 * *$ & 0,14 \\
\hline \multicolumn{7}{|l|}{ Makroindikatoren } \\
\hline Erwerbsquote 55-64 & & & & & 1,00 & 0,00 \\
\hline Civil Liberties Index & & & & & $1,08 *$ & 0,04 \\
\hline Anteil Sozialausgaben am BIP & & & & & $1,09^{+}$ & 0,04 \\
\hline$\sigma_{u}$ & $0,75 * *$ & 0,16 & $0,63 * *$ & 0,13 & $0,33 * *$ & 0,07 \\
\hline$\rho$ & 0,14 & 0,05 & 0,10 & 0,04 & 0,03 & 0,01 \\
\hline$(\mathrm{N}=27.266)$ & & & & & & \\
\hline
\end{tabular}

Signifikanzniveaus: ${ }^{+} 10 \%, * 5 \%, * * 1 \%$

${ }^{a}$ Referenzkategorie

Quelle: SHARE 2004 (Release 2.0.1), eigene Berechnungen. 
Tabelle 3: Ergebnisse der logistischen Mehrebenenmodelle für die abhängige Variable ,Netzwerkhilfe'

\begin{tabular}{|c|c|c|c|c|c|c|}
\hline \multirow[b]{2}{*}{ Variablen } & \multicolumn{2}{|c|}{ Modell 1} & \multicolumn{2}{|c|}{ Modell 2} & \multicolumn{2}{|c|}{ Modell 3} \\
\hline & OR & s.e. & OR & s.e. & OR & s.e. \\
\hline \multicolumn{7}{|l|}{ Demographische Merkmale } \\
\hline Geschlecht weiblich & & & $1,09 * *$ & 0,03 & $1,09 * *$ & 0,03 \\
\hline Alter $50-64^{\mathrm{a}}$ & & & & & & \\
\hline Alter $65-74$ & & & $0,61 * *$ & 0,02 & $0,61 * *$ & 0,02 \\
\hline Alter $75+$ & & & $0,28 * *$ & 0,01 & $0,28 * *$ & 0,01 \\
\hline Mit Partner lebend & & & 1,00 & 0,03 & 1,00 & 0,03 \\
\hline \multicolumn{7}{|l|}{ Sozio-ökonomische Merkmale } \\
\hline Erwerbstätig & & & $0,92^{+}$ & 0,04 & $0,92^{+}$ & 0,04 \\
\hline Nicht erwerbstätig & & & $0,91^{+}$ & 0,04 & $0,91^{+}$ & 0,04 \\
\hline Im Ruhestand ${ }^{\mathrm{a}}$ & & & & & & \\
\hline Niedriger Bildungsabschluss ${ }^{\mathrm{a}}$ & & & & & & \\
\hline Mittlerer Bildungsabschluss & & & $1,23 * *$ & 0,04 & $1,22 * *$ & 0,04 \\
\hline Hoher Bildungsabschluss & & & $1,33 * *$ & 0,05 & $1,32 * *$ & 0,05 \\
\hline \multicolumn{7}{|l|}{ Gesundheitsmerkmale } \\
\hline Subjektive Gesundheit & & & $0,65 * *$ & 0,02 & $0,65 * *$ & 0,02 \\
\hline $2+$ chronische Krankheiten & & & $1,17 * *$ & 0,04 & $1,17 * *$ & 0,04 \\
\hline Depressionssymptome & & & $1,02^{+}$ & 0,04 & 1,02 & 0,04 \\
\hline \multicolumn{7}{|l|}{ Dimensionen informeller Arbeit } \\
\hline Ehrenamt & & & $1,81 * *$ & 0,07 & $1,81 * *$ & 0,07 \\
\hline Pflege & & & $2,94 * *$ & 0,15 & $2,94 * *$ & 0,15 \\
\hline \multicolumn{7}{|l|}{ Makroindikatoren } \\
\hline Erwerbsquote 55-64 & & & & & 1,00 & 0,00 \\
\hline Civil Liberties Index & & & & & $1,04^{+}$ & 0,02 \\
\hline Anteil Sozialausgaben am BIP & & & & & $1,12 * *$ & 0,03 \\
\hline$\sigma_{u}$ & $0,62 * *$ & 0,13 & $0,54 * *$ & 0,11 & $0,20 * *$ & 0,04 \\
\hline$\rho$ & 0,10 & 0,04 & 0,08 & 0,03 & 0,01 & 0,00 \\
\hline$(\mathrm{N}=27.266)$ & & & & & & \\
\hline
\end{tabular}

Signifikanzniveaus: ${ }^{+} 10 \%, * 5 \%, * * 1 \%$

${ }^{a}$ Referenzkategorie

Quelle: SHARE 2004 (Release 2.0.1), eigene Berechnungen. 
Tabelle 4: Ergebnisse der logistischen Mehrebenenmodelle

für die abhängige Variable ,Pflege'

\begin{tabular}{|c|c|c|c|c|c|c|}
\hline \multirow[b]{2}{*}{ Variablen } & \multicolumn{2}{|c|}{ Modell 1} & \multicolumn{2}{|c|}{ Modell 2} & \multicolumn{2}{|c|}{ Modell 3} \\
\hline & OR & s.e. & OR & s.e. & OR & s.e. \\
\hline \multicolumn{7}{|l|}{ Demographische Merkmale } \\
\hline Geschlecht weiblich & & & $1,50 * *$ & 0,08 & $1,50 * *$ & 0,08 \\
\hline Alter $50-64^{\mathrm{a}}$ & & & & & & \\
\hline Alter $65-74$ & & & $0,77 * *$ & 0,05 & $0,77 * *$ & 0,05 \\
\hline Alter $75+$ & & & $0,72 * *$ & 0,06 & $0,72 * *$ & 0,06 \\
\hline Mit Partner lebend & & & 1,08 & 0,06 & 1,08 & 0,06 \\
\hline \multicolumn{7}{|l|}{ Sozio-ökonomische Merkmale } \\
\hline Erwerbstätig & & & 0,90 & 0,06 & 0,90 & 0,06 \\
\hline Nicht erwerbstätig & & & 0,96 & 0,07 & 0,97 & 0,07 \\
\hline Im Ruhestand ${ }^{\mathrm{a}}$ & & & & & & \\
\hline Niedriger Bildungsabschluss ${ }^{\mathrm{a}}$ & & & & & & \\
\hline Mittlerer Bildungsabschluss & & & $1,31 * *$ & 0,08 & $1,31 * *$ & 0,08 \\
\hline Hoher Bildungsabschluss & & & $1,40 * *$ & 0,09 & $1,39 * *$ & 0,09 \\
\hline \multicolumn{7}{|l|}{ Gesundheitsmerkmale } \\
\hline Subjektive Gesundheit & & & $0,89^{+}$ & 0,05 & $0,89^{+}$ & 0,05 \\
\hline $2+$ chronische Krankheiten & & & $1,05 *$ & 0,06 & 1,05 & 0,06 \\
\hline Depressionssymptome & & & $1,53 * *$ & 0,09 & $1,53 * *$ & 0,09 \\
\hline \multicolumn{7}{|l|}{ Dimensionen informeller Arbeit } \\
\hline Ehrenamt & & & $2,34 * *$ & 0,14 & $2,33 * *$ & 0,14 \\
\hline Netzwerkhilfe & & & $2,93 * *$ & 0,15 & $2,91 * *$ & 0,15 \\
\hline \multicolumn{7}{|l|}{ Makroindikatoren } \\
\hline Erwerbsquote 55-64 & & & & & 0,99 & 0,00 \\
\hline Civil Liberties Index & & & & & 0,97 & 0,02 \\
\hline Anteil Sozialausgaben am BIP & & & & & $1,06 *$ & 0,03 \\
\hline$\sigma_{u}$ & $0,35 * *$ & 0,08 & $0,23 * *$ & 0,06 & $0,19 * *$ & 0,05 \\
\hline$\rho$ & 0,03 & 0,01 & 0,01 & 0,00 & 0,01 & 0,00 \\
\hline$(\mathrm{N}=27.266)$ & & & & & & \\
\hline
\end{tabular}

Signifikanzniveaus: ${ }^{+} 10 \%, * 5 \%, * * 1 \%$

${ }^{a}$ Referenzkategorie

Quelle: SHARE 2004 (Release 2.0.1), eigene Berechnungen. 


\section{Discussion Paper Series}

Mannheim Research Institute for the Economics of Aging Universität Mannheim

To order copies, please direct your request to the author of the title in question.

\begin{tabular}{|c|c|c|c|}
\hline Nr. & Autoren & Titel & Jahr \\
\hline $140-07$ & Hendrik Jürges & $\begin{array}{l}\text { Health inequalities by education, income, and } \\
\text { wealth: a comparison of } 11 \text { European countries } \\
\text { and the US }\end{array}$ & 07 \\
\hline $141-07$ & Hendrik Jürges & $\begin{array}{l}\text { Healthy minds in healthy bodies. An } \\
\text { international comparison of education-related } \\
\text { inequality in physical health among older adults }\end{array}$ & 07 \\
\hline $142-07$ & $\begin{array}{l}\text { Karsten Hank, } \\
\text { Stephanie Stuck }\end{array}$ & $\begin{array}{l}\text { Volunteer Work, Informal Help, and Care among } \\
\text { the 50+ in Europe: Further Evidence for 'Linked' } \\
\text { Productive Activities at Older Ages }\end{array}$ & 07 \\
\hline $143-07$ & Jürgen Maurer & $\begin{array}{l}\text { Assessing Horizontal Equity in Medication } \\
\text { Treatment Among Elderly Mexicans: Which } \\
\text { Socioeconomic Determinants Matter Most? }\end{array}$ & 07 \\
\hline $144-07$ & Jürgen Maurer & $\begin{array}{l}\text { Socioeconomic and Health Determinants of } \\
\text { Health Care Utilization Among Elderly } \\
\text { Europeans: A Semiparametric Assessment of } \\
\text { Equity, Intensity and Responsiveness for Ten } \\
\text { European Countries }\end{array}$ & 07 \\
\hline $145-07$ & Jürgen Maurer & $\begin{array}{l}\text { Modelling socioeconomic and health } \\
\text { determinants of health care use: A } \\
\text { semiparametric approach }\end{array}$ & 07 \\
\hline $146-07$ & $\begin{array}{l}\text { Jérome Adda, James } \\
\text { Banks, Hans-Martin } \\
\text { von Gaudecker }\end{array}$ & $\begin{array}{l}\text { The Impact of Income Shocks on Health: } \\
\text { Evidence from Cohort Data }\end{array}$ & 07 \\
\hline $147-07$ & $\begin{array}{l}\text { Klaus Jäger, } \\
\text { Wolfgang Kuhle }\end{array}$ & $\begin{array}{l}\text { The Optimum Growth Rate for Population } \\
\text { Reconsidered }\end{array}$ & 07 \\
\hline $148-07$ & $\begin{array}{l}\text { Axel Börsch-Supan, } \\
\text { Ismail Düzgün, } \\
\text { Matthias Weiss }\end{array}$ & $\begin{array}{l}\text { Age and Productivity in Work Teams: Evidence } \\
\text { from the Assembly Line }\end{array}$ & 07 \\
\hline $149-07$ & Matthias Weiss & Sick Leave and the Composition of Work Teams & 07 \\
\hline $150-07$ & Matthias Weiss & Are Age-Diverse Work Teams Better? & 07 \\
\hline $151-07$ & $\begin{array}{l}\text { Alexander Ludwig, } \\
\text { Thomas Schelkle, } \\
\text { Edgar Vogel }\end{array}$ & $\begin{array}{l}\text { Demographic Change, Human Capital and } \\
\text { Endogenous Growth }\end{array}$ & 07 \\
\hline $152-07$ & $\begin{array}{l}\text { Karsten Hank } \\
\text { Stephanie Stuck }\end{array}$ & $\begin{array}{l}\text { Gesellschaftliche Determinanten produktiven } \\
\text { Alterns in Europa }\end{array}$ & 07 \\
\hline
\end{tabular}

